\title{
Current perspective on the role of the interleukin-23/interleukin- 17 axis in inflammation and disease (chronic arthritis and psoriasis)
}

This article was published in the following Dove Press journal:

ImmunoTargets and Therapy

I October 2015

Number of times this article has been viewed

\section{Alberto Cauli \\ Matteo Piga \\ Alberto Floris \\ Alessandro Mathieu}

Rheumatology Unit, Department of Medical Sciences, Policlinico of the University of Cagliari, Monserrato, Cagliari, Italy
Correspondence: Alberto Cauli Rheumatology Unit, Department of Medical Sciences, Policlinico of the University of Cagliari, ss554, Monserrato, 090 I 42 Cagliari, Italy

Tel +390706754229

Fax +390706754229

Email cauli@medicina.unica.it
Abstract: TH17 is a lymphocyte subset, which is characterized by its polarization to secrete interleukin (IL)-17. IL-23 is the pivotal mediator responsible for TH17 differentiation and the IL-23/IL-17 axis has been strongly implicated in the pathogenesis of several immune mediated diseases, in particular chronic arthritis and skin psoriasis. This review will summarize the basic immunology and the new monoclonal antibodies, which antagonize this pathway allowing a new therapeutic approach.

Keywords: TH17, IL-17, IL-23, psoriasis, psoriatic arthritis, ankylosing spondylitis

\section{A T-cell subset on the stage: $\mathrm{THI} 7$}

TH17 is a CD3+/CD4+ T lymphocyte subset, which is characterized by its polarization to secrete interleukin (IL)-17. The inflammatory cytokine IL-17 has been implicated in self-protection versus protozoa, bacteria, and fungi. ${ }^{1}$ The first description of IL-17 production by CD4 $\mathrm{T}$ was in $1995,{ }^{2}$ but only in the last decade the concept of a separate $\mathrm{CD} 4+\mathrm{T}$ subset producing IL-17, distinct from TH1 and TH2 subsets, has strongly emerged in the scientific literature. ${ }^{3,4}$ The old dichotomy in $\mathrm{T}$ lymphocyte classification pictured a scenario where antigen stimulation of naïve CD4+ T cells could determine the emergence either of TH1 lymphocytes secreting interferon- $\gamma($ IFN- $\gamma$ ) implicated in the mechanisms of protection to intracellular pathogens (also playing a major role in autoimmunity), or TH2 lymphocytes producing cytokines such as IL-4, IL-5, IL-6, and IL-13, implicated in the immune processes of defense versus extracellular organisms, humoral immunity, and allergy.

The development of the TH1 subset is triggered by various stimuli, in particular by the cytokine IL-12; an early innate immune reaction driven by natural killer cells is also able to activate the T-bet transcription factor through signal transducer and activator of transcription 1, thus enhancing response to IL-12; this is of importance because the therapeutic monoclonal antibody Ustekinumab is directed toward the IL-12p40 subunit that is shared by both IL-12 and IL-23, and therefore it affects both TH1 and TH17 subsets. The pivotal cytokine IL-23, together with other mediators such as IL-1, tumor necrosis factor alpha (TNF- $\alpha$ ), and IL-6, is fundamental in directing naïve CD4+ T cells toward the TH17 subset. The possible role of transforming growth factor- $\beta$ in TH17 differentiation in the human immune system is still controversial, while this has been clearly demonstrated in mice models. ${ }^{5}$ In this regard it is relevant that, in naïve T lymphocytes, transforming growth factor- $\beta$ and IL-6 induce retinoic acid orphan receptors (RORgt and RORa), triggering the upregulation of IL-23 receptor 
(IL-23R) and therefore easing the polarization to TH17. ${ }^{6}$ The dimeric cytokine IL-23 is included in the IL-12 family and is composed of the two subunits IL-12p40 and IL-23p19, while IL-12 is composed of IL-12p35 and IL-12p40 subunits (IL12p70). ${ }^{7}$ This dimeric molecule structure has to be considered in drug design for the pharmacological and therapeutic consequences. The structural similarities of these two cytokines are also reflected in their receptors, which share a common subunit. The IL-12 receptor is constituted by IL12Rb1 in association with the IL12Rb2 subunit while the IL-23 receptor is composed of the IL12Rb1 and IL-23R subunits. All the data so far available suggest that IL-23 is the pivotal mediator implicated in the differentiation and maturation of the TH17 subset, as well as in the stimuli for IL-17 secretion. ${ }^{8}$

The TH17 subset is modulated by distinct mediators and mechanisms: IFN- $\gamma$ and IL-4 downregulate IL-17 secretion, ${ }^{3,4,9}$ IL-2 inhibits TH17 cell maturation by downregulating ROR $\gamma \delta,{ }^{10}$ IL-27 (an antigen-presenting cell-derived cytokine part of the IL-12 family) inhibits IL-17 synthesis by inducing IL-10 secretion from Treg cells. ${ }^{11}$ In humans, TH17 cells secrete IL-17A and IL-17F but other cells are also capable of producing IL-17, namely neutrophils, natural killer cells, CD8+ lymphocytes, and $\gamma \delta \mathrm{T}$ cells, which can produce other IL-17 family cytokines. ${ }^{12}$

In this regard, it is noteworthy that neutrophils accumulate in the upper layer of psoriatic skin, giving rise to the formation of Munro's microabscesses, ${ }^{13}$ and that neutrophils depletion by means of monoclonal antibodies has been shown to reduce epidermal thickening in mice. ${ }^{14}$ Furthermore, innate lymphoid cells such as $\gamma \delta \mathrm{T}$ cells are elevated in psoriatic skin and constitutively express IL-23, ${ }^{15}$ contributing to aberrant keratinocyte differentiation, acanthosis and hyperplasia, hyperkeratosis and parakeratosis. It is also noteworthy that innate lymphoid cells producing IL-17 and IL-22 are expanded in the gut, in the peripheral blood, synovial fluid, and bone marrow of patients with ankylosing spondylitis (AS), supporting the existence of a multiorgan active axis in the spondyloarthritis ( $\mathrm{SpA})$ spectrum of diseases. ${ }^{16}$

TH17 lymphocytes are also capable of producing other cytokines such as TNF- $\alpha$, IL- $6,{ }^{17}$ and IL-22. ${ }^{18}$ In the scenario of psoriasis pathogenesis, it has to be underlined that IL-22 has been shown to stimulate epidermal hyperplasia and dermal inflammation. ${ }^{19}$ Many cells have receptors for and are targets of IL-17, among them monocytes and macrophages, osteoblasts, fibroblasts, endothelial and epithelial cells. IL-17 biological action includes the stimuli to the production of the pro-inflammatory cytokines IL-6, TNF- $\alpha$, IL-1 $\beta$, and IL-8, as well as the induction of homing receptors and chemokines expression, the upregulation of colony stimulating factors and matrix metalloprotease expression. ${ }^{12}$

\section{IL-23 and the THI 7 subset in chronic arthritis}

For many years rheumatoid arthritis (RA), ${ }^{20,12}$ psoriasis, and psoriatic arthritis (PsA), ${ }^{21,22}$ as well as AS, ${ }^{23,24}$ were considered to be pathological processes driven by the TH1 subset, and IFN- $\gamma$ and IL-2 were supposed to be the leading mediators in the generation of the inflammatory cascade. In the recent past, emerging data have underlined the role of the TH17 subset and IL-17 in the pathological mechanisms leading to chronic arthritis such as PsA, AS, and RA, as well as inflammatory bowel diseases (IBDs) and skin psoriasis.

Strong evidence links these diseases to the TH17 subset; first of all the data arising from genome-wide association studies and genetic studies. The susceptibility to skin psoriasis and PsA is associated with alleles of the $I L 12 B$ and IL23R. ${ }^{25,26}$ Moreover, AS and Crohn's disease, belonging to the spectrum of $\mathrm{SpA}$ together with PsA, are also linked to IL23R alleles. ${ }^{27,28}$ This common genetic background easily suggests a common inflammatory pathway, which involves the receptor of IL-23. More data support the importance of the IL-23/TH17 axis in these diseases; IL23p19 and p40 (shared by IL-12p70 and IL-23 cytokines) subunits, but not IL-12p35 (present in IL-12 only), have been strongly detected in involved psoriatic skin as compared with non-lesional skin. ${ }^{29}$ Furthermore, serum levels of IL-12/23 p40 subunit have been detected at significantly higher levels in PsA patients compared with healthy controls. ${ }^{30}$ We have previously underlined the importance of the IL-23 cytokine and its receptor in TH17 maturation and function; the experimental data summarized support a primary role of the TH17 subset in SpA and skin psoriasis.

Several data also support the possible involvement of the IL-23/TH17 axis even in RA; both IL-17 and IL-23 have been detected in the synovial membrane, in the synovial fluid as well as in peripheral blood serum; ${ }^{31}$ moreover, IL-23 expression has been correlated with the expression of receptor activator of nuclear factor kappa $B$ ligand at the mRNA level. ${ }^{32} \mathrm{IL}-17$ and TNF- $\alpha$ are able to induce receptor activator of nuclear factor kappa B ligand, which activates osteoclasts that are responsible for erosions at sites of articular inflammation. ${ }^{12}$ These data provide support to the concept that the IL-23/IL-17 axis is involved in the mechanisms of bone erosion and joint destruction. As previously outlined, IBDs are part of the SpA spectrum of diseases and therefore it is of importance to note the report of an overexpression of IL-17 
in the gut tissue and sera of these patients, ${ }^{33}$ as well as the finding of an increased number of TH17 cells in gut biopsies from Crohn's patients. ${ }^{34}$

The SpA spectrum of disease has been known for a long time to be associated with the HLA antigen B27. Genome-wide association studies have more recently demonstrated the association with IL-23R and the data previously summarized strongly underline the role of the TH17 subset. In 2011, the Oxford group of Bownes et al nicely summarized the evidence which arose from experimental work done in their laboratory, as well as data from the scientific community, and have linked together the HLA-B27 association with KIR receptors and the IL-23/TH17 axis. B27 is a hetero-trimeric molecule that has the tendency to dissociate from the bound peptide and the $\beta 2$ microglobulin component. This dissociation allows free heavy chains to form $\beta 2$ microglobulin-free heavy chain homodimers (B27 ${ }_{2}$, which are able to stimulate the proliferation, survival and IL-17 production of KIR3DL2+CD4+Th-17 cells; it has been also demonstrated that these effects are mediated by the KIR3DL2/B27 ${ }_{2}$ pair interaction. ${ }^{35,36}$ It is noteworthy that B27 ${ }_{2}$ responsive IL-17 producing CD4+ T cells were IL-23R positive and were also able to produce TNF- $\alpha$ and/or IFN- $\gamma$. Cells of the $\mathrm{T}$ lymphocyte lineage, showing an overlapping TH17 and TH1 phenotype (plasticity) can be detected in SpA patients widening the scenario of their possible role in disease as well as in physiologic functions.

\section{New emerging drugs that interfere with the IL-23/IL- 17 axis}

The possibility that antagonism of this pathway may allow the key approach in the control of disease activity as well as disease damage in chronic arthritis and in other immunemediated inflammatory diseases has been tested "in vivo" by means of several new biologic drugs, which target the IL-23/IL-17 axis, as summarized in Table 1. This review will focus on the three compounds, which have been more extensively studied at present, Ustekinumab, Secukinumab, and Brodalumab.

Ustekinumab is an IgG human monoclonal antibody that recognizes the p40 subunit of both IL-12 and IL-23 cytokines, therefore influencing the activity of the TH1 and TH17 pathways. Ustekinumab has been assessed in two Phase III trials in psoriasis; in the first study, patients were treated with $45 \mathrm{mg}, 90 \mathrm{mg}$, or placebo at baseline, ${ }^{37}$ after 4 weeks and then every 12 weeks. They achieved a PASI75 at week 12 of $67.1 \%, 66.4 \%$, and $3.1 \%$, respectively; results from the second study were comparable. ${ }^{38}$ Other measures of response
Table I Monoclonal antibodies developed for use in human diseases which interfere with the interleukin (IL)-23/IL-I 7 axis

IL- 17 inhibitors

Secukinumab

Ixekizumab

Brodalumab

IL-23 inhibitors

Guselkumab

Tildrakizumab

IL- 12 and IL-23 inhibitors

Ustekinumab

included nail disease and quality of life, which also showed clear improvements. No serious side effects were reported in these two trials.

Ustekinumab was also tested in two Phase III trials in PsA. In PSUMMIT 1, patients with an inadequate response to methotrexate were randomized to receive the same dose regimen as in the psoriasis trials, ${ }^{39}$ the primary end point was ACR20 at week 24 that resulted in $42.4 \%$ and $49.5 \%$ in the two active drug arms compared with $22.8 \%$ in placebo. Other domains of investigation included enthesitis, dactylitis, skin, nail disease, quality of life, and function, where all results were improved. Similar side effects incidence was noted in the three groups, without opportunistic infections or major cardiovascular events. PSUMMIT 2 trial differed in the inclusion criteria of patients that allowed the recruitment of patients previously exposed to TNF- $\alpha$ antagonists, showing similar significant positive response of the patients in the active arms. Furthermore, pooled radiographic data from the two studies showed an inhibition of structural damage. ${ }^{40}$ Promising preliminary results have been also obtained by the administration of Ustekinumab in AS patients, while no formal data are available on the use of Ustekinumab in RA. ${ }^{41}$

Secukinumab is an IgG1k human monoclonal antibody that recognizes IL-17A. Two Phase III studies have been performed in psoriasis (ERASURE and FIXTURE). In the first one, patients were randomized to receive subcutaneous Secukinumab at doses of 300 or $150 \mathrm{mg}$ once weekly for 5 weeks and then every 4 weeks compared with placebo. ${ }^{42}$ PASI75 was reached at week 12 by $81.6 \%, 71.6 \%$, and $4.5 \%$ of patients, respectively. In the FIXTURE study (which compared Secukinumab versus Etanercept) PASI75 was reached by $77.1 \%, 67.0 \%, 44.0 \%$, and $4.9 \%$ of patients on $300 \mathrm{mg}$ or $150 \mathrm{mg}$ of Secukinumab, $50 \mathrm{mg}$ twice weekly of Etanercept or placebo, respectively. Secukinumab also demonstrated to be effective in nail disease, itch, and quality of life; serious side effects were infrequent and comparable among all arms. Candida infections were reported as mild-moderate in $4.7 \%$, 
$2.3 \%$, and $1.2 \%$ of patients in the $300 \mathrm{mg}$ or $150 \mathrm{mg}$ Secukinumab or Etanercept groups, respectively.

In PsA, Secukinumab has been studied in two Phase III trials (FUTURE 1 and 2 trials). In FUTURE 1, patients were randomized to receive an intravenous loading dose of Secukinumab $10 \mathrm{mg} / \mathrm{kg}$ at baseline, ${ }^{43}$ weeks 2 and 4 and then $150 \mathrm{mg}$ or $75 \mathrm{mg}$ every 4 weeks starting from week 8 compared with placebo. At week 24, ACR20 was $50.0 \%, 50.5 \%$, and $17.3 \%$, Secukinumab $150 \mathrm{mg}, 75 \mathrm{mg}$, or placebo, respectively. Other domains investigated included skin disease, dactylitis, enthesitis, function, quality of life, and inhibition of X-ray progression of damage, which all resulted statistically different from placebo. In the FUTURE 2 trial, patients did not receive an intravenous loading dose; ${ }^{44}$ results confirmed the efficacy in the same domains as in FUTURE 1 trial. Overall adverse effects in PsA patients were similar to psoriatic patients; the total infection rate was slightly higher in the Secukinumab arm compared with placebo, without opportunistic or tuberculosis infections.

Two Phase III trials have been also performed in AS: MEASURE 1 and MEASURE 2. In the first trial, patients were randomized in three arms after a $10 \mathrm{mg} / \mathrm{kg}$ weekly loading dose of Secukinumab for the first 4 weeks, followed by $75 \mathrm{mg}$ or $150 \mathrm{mg}$ or placebo subcutaneously every 4 weeks. ${ }^{45}$ The primary end point was ASAS20 response at week 16, which was reached by $59.7 \%$ and $60.8 \%$ of patients in the active arms and $28.7 \%$ in the placebo group, respectively. In the MEASURE 2 trial, patients did not receive the loading dose; ${ }^{46}$ the ASAS20 responses were $41.1 \%, 61.1 \%$, and $28.4 \%$, respectively. Side effects were in line with the previous studies.

It is remarkable that a Phase II study performed in RA was not able to separate the active drug arms compared with placebo in terms of ACR20 response, ${ }^{37}$ although Secukinumabtreated patients showed statistically lower DAS28 scores and lower high sensitivity C-reactive protein values.

Brodalumab is a human monoclonal antibody that blocks IL17A receptor, therefore inhibiting IL-17 signaling. Brodalumab has been proved to be effective in a Phase II study in psoriasis; ${ }^{47}$ patients were treated with 70, 140, 210 , or $280 \mathrm{mg}$ of Brodalumab subcutaneously and PASI75 response rates at 12 weeks were $45 \%, 85 \%, 86.3 \%$, and $76 \%$, respectively, compared with $16 \%$ of placebo controls. A Phase II study was also performed in PsA, aiming to an ACR20 response primary end point at 12 weeks; $37 \%$ and $39 \%$ of patients treated in the 140 and $280 \mathrm{mg}$ active drug arms reached the primary end point compared with $18 \%$ in the placebo arm. ${ }^{48}$ Brodalumab has been studied also in RA, without benefit. In a Phase I ascending dose study, separation of active drug-treated patients compared with placebo was not demonstrated; ${ }^{49}$ furthermore in a Phase II study performed in methotrexate inadequate responders similar negative results were obtained. ${ }^{50}$

In this study, Pavelka et al reported a clear lack of therapeutic effect of IL-17 antagonism in RA, and the authors' conclusion was that there is no reason to pursue further evaluation of Brodalumab in this disease. Although this is a negative study the lesson that arises from this publication is of value. Together with previously published data for Secukinumab, an anti-IL-17A antibody, the results of this trial strongly indicate that the IL-17 pathway is not an appropriate therapeutic target for RA while it is successful in PsA. This evidence is instructive and may help in clarifying the important pathogenic differences that characterize RA compared with PsA; similar disease in some features, so different in others.

Marketed or developing drugs that inhibit IL-17, IL-23, or the IL-12/IL-23 shared subunit IL-12p40 have demonstrated significant benefit in $\mathrm{SpA}$ and skin psoriasis. Further studies and post-marketing data will reveal the best approach in order to efficiently interfere in the pathogenetic mechanisms of TH17-mediated diseases.

\section{Conclusion}

The precise pathogenesis of chronic arthritis, skin psoriasis, and IBD, as well as the role of the TH17 subset in their inflammatory milieu, needs further in depth research. Experimental clinical work performed so far in patients with these diseases, thanks to the availability of new biologic compounds targeting the IL-23/IL-17 axis, suggests the important features that differentiate SpA and psoriasis from IBD and RA. These differences are reflected in the response to treatment and therefore deserve our greatest attention.

The availability of new effective drugs that target different mediators and inflammatory pathways is of great importance in order to increase opportunities for the physician to impact in disease activity and progression for the benefit of the patients.

\section{Disclosure}

The authors report no conflicts of interest in this work.

\section{References}

1. Lubberts E. Th17 cytokines and arthritis. Semin Immunopathol. 2010;32:43-53.

2. Yao Z, Painter SL, Fanslow WC, et al. Human IL-17: a novel cytokine derived from T cells. J Immunol. 1995;155:5483-5486. 
3. Harrington LE, Hatton RD, Mangan PR, et al. Interleukin 17-producing CD4+ effector T cells develop via a lineage distinct from the T helper type 1 and 2 lineages. Nat Immunol. 2005;6:1123-1132.

4. Park H, Li Z, Yang XO, et al. A distinct lineage of CD4 T cells regulates tissue inflammation by producing interleukin 17. Nat Immunol. 2005;6:1133-1141.

5. McGeachy MJ, Bak-Jensen KS, Chen Y, et al. TGF-beta and IL-6 drive the production of IL-17 and IL-10 by T cells and restrain T(H)-17 cellmediated pathology. Nat Immunol. 2007;8:1390-1397.

6. Yang XO, Pappu BP, Nurieva R, et al. T helper 17 lineage differentiation is programmed by orphan nuclear receptors ROR alpha and ROR gamma. Immunity. 2008;28:29-39.

7. Langrish CL, McKenzie BS, Wilson NJ, et al. IL-12 and IL-23: master regulators of innate and adaptive immunity. Immunol Rev. 2004;202: 96-105.

8. Kastelein RA, Hunter CA, Cua DJ. Discovery and biology of IL-23 and IL-27: related but functionally distinct regulators of inflammation. Annu Rev Immunol. 2007;25:221-242.

9. Hoeve MA, Savage ND, de Boer T, et al. Divergent effects of IL-12 and IL-23 on the production of IL-17 by human T cells. Eur J Immunol. 2006;36:661-670.

10. Laurence A, Tato CM, Davidson TS, et al. Interleukin-2 signaling via STAT5 constrains T helper 17 cell generation. Immunity. 2007;26: 371-381.

11. Awasthi A, Carrier Y, Peron JP, et al. A dominant function for interleukin 27 in generating interleukin 10-producing anti-inflammatory $\mathrm{T}$ cells. Nat Immunol. 2007;8:1380-1389.

12. Weaver CT, Hatton RD, Mangan PR, Harrington LE. IL-17 family cytokines and the expanding diversity of effector T cell lineages. Annu Rev Immunol. 2007;25:821-852.

13. Steffen C. William John Munro and Munro's abscess, and Franz Kogoj and Kogoj's spongiform pustule. Am J Dermatopathol. 2002;24:364-368.

14. Schon M, Denzer D, Kubitza RC, Ruzicka T, Schon MP. Critical role of neutrophils for the generation of psoriasiform skin lesions in flaky skin mice. J Invest Dermatol. 2000;114:976-983.

15. Cai Y, Shen X, Ding C, et al. Pivotal role of dermal IL-17-producing gammadelta T cells in skin inflammation. Immunity. 2011;35:596-610.

16. Ciccia F, Guggino G, Rizzo A, et al. Type 3 innate lymphoid cells producing IL-17 and IL-22 are expanded in the gut, in the peripheral blood, synovial fluid and bone marrow of patients with ankylosing spondylitis. Ann Rheum Dis. 2015; pii:annrheumdis-2014-206323.

17. Yen D, Cheung J, Scheerens H, et al. IL-23 is essential for T cellmediated colitis and promotes inflammation via IL-17 and IL-6. J Clin Invest. 2006;116:1310-1316.

18. Liang SC, Tan XY, Luxenberg DP, et al. Interleukin (IL)-22 and IL-17 are coexpressed by Th17 cells and cooperatively enhance expression of antimicrobial peptides. $J$ Exp Med. 2006;203:2271-2279.

19. Ma HL, Liang S, Li J, et al. IL-22 is required for Th17 cell-mediated pathology in a mouse model of psoriasis-like skin inflammation. J Clin Invest. 2008;118:597-607.

20. Cauli A, Yanni G, Panayi GS. Interleukin-1, interleukin-1 receptor antagonist and macrophage populations in rheumatoid arthritis synovial membrane. Br J Rheumatol. 1997;36:935-940.

21. Cauli A, Pitzalis C, Yanni G, Hawad M, Panayi GS. CD1 positive antigen presenting cells in psoriatic and rheumatoid arthritis. Rheumatology. 2000;6:666-673.

22. Pitzalis C, Cauli A, Pipitone N, et al. Cutaneous lymphocyte antigenpositive $\mathrm{T}$ lymphocytes preferentially migrate to the skin but not to the joint in psoriatic arthritis. Arthritis Rheum. 1996;39:137-145.

23. Cauli A, Dessole G, Fiorillo MT, A et al. Increased level of HLA-B27 expression in ankylosing spondylitis patients compared with healthy HLA-B27-positive subjects: a possible further susceptibility factor for the development of disease. Rheumatology. 2002;41:1375-1379.

24. Cauli A, Dessole G, Vacca A, et al. Susceptibility to ankylosing spondylitis but not disease outcome is influenced by the level of HLA-B27 expression, which shows moderate variability over time. Scand $J$ Rheumatol. 2012;41:214-218.
25. Liu Y, Helms C, Liao W, et al. A genome-wide association study of psoriasis and psoriatic arthritis identifies new disease loci. PLoS Genet. 2008:4:e1000041.

26. Nair RP, Ruether A, Stuart PE, et al. Polymorphisms of the IL12B and IL23R genes are associated with psoriasis. $J$ Invest Dermatol. 2008;128:1653-1661.

27. Wellcome Trust Case Control Consortium, Australo-Anglo-American Spondylitis Consortium (TASC), Burton PR, et al. Association scan of 14,500 nonsynonymous SNPs in four diseases identifies autoimmunity variants. Nat Genet. 2007;39:1329-1337.

28. Duerr RH, Taylor KD, Brant SR, et al. A genome-wide association study identifies IL23R as an inflammatory bowel disease gene. Science. 2006;314:1461-1463.

29. Lee E, Trepicchio WL, Oestreicher JL, et al. Increased expression of interleukin 23 p19 and p40 in lesional skin of patients with psoriasis vulgaris. J Exp Med. 2004;199:125-130.

30. Szodoray P, Alex P, Chappell-Woodward CM, et al. Circulating cytokines in Norwegian patients with psoriatic arthritis determined by a multiplex cytokine array system. Rheumatology. 2007;46:417-425.

31. Kotake S, Udagawa N, Takahashi N, et al. IL-17 in synovial fluids from patients with rheumatoid arthritis is a potent stimulator of osteoclastogenesis. J Clin Invest. 1999;103:1345-1352.

32. Sato K, Suematsu A, Okamoto K, et al. Th17 functions as an osteoclastogenic helper $\mathrm{T}$ cell subset that links $\mathrm{T}$ cell activation and bone destruction. J Exp Med. 2006;203:2673-2682.

33. Fujino S, Andoh A, Bamba S, et al. Increased expression of interleukin 17 ininflammatory bowel disease. Gut. 2003;52:65-70.

34. Annunziato F, Cosmi L, Santarlasci V, et al. Phenotypic and functional features of human Th17 cells. J Exp Med. 2007;204:1849-1861.

35. Bowness P, Ridley A, Shaw J, et al. Th17 cells expressing KIR3DL2 ${ }^{+}$ and responsive to HLA-B27 homodimers are increased in ankylosing spondylitis. J Immunol. 2011;186:2672-2680.

36. Cauli A, Shaw J, Giles J, et al. The arthritis-associated HLA-B*27:05 allele forms more cell surface B27 dimer and free heavy chain ligands for KIR3DL2 than HLA-B*27:09. Rheumatology. 2013;52: 1952-1962.

37. Genovese MC, Durez P, Richards HB, et al. Efficacy and safety of secukinumab in patients with rheumatoid arthritis: a phase II, dosefinding, doubleblind, randomised, placebo controlled study. Ann Rheum Dis. 2013;72:863-869.

38. Papp KA, Langley RG, Lebwohl M, et al. Efficacy and safety of ustekinumab, a human interleukin-12/23 monoclonal antibody, in patients with psoriasis: 52-week results from a randomised, double-blind, placebocontrolled trial (PHOENIX 2). Lancet. 2008;371:1675-1684.

39. McInnes IB, Kavanaugh A, Gottlieb AB, et al. Efficacy and safety of ustekinumab in patients with active psoriatic arthritis: 1 year results of the phase 3, multicentre, double-blind, placebo-controlled PSUMMIT 1 trial. Lancet. 2013;382:780-789.

40. Kavanaugh A, Ritchlin C, Rahman P, et al. Ustekinumab, an anti-IL$12 / 23$ p40 monoclonal antibody, inhibits radiographic progression in patients with active psoriatic arthritis: results of an integrated analysis of radiographic data from the phase 3 , multicentre, randomised, doubleblind, placebo-controlled PSUMMIT-1 and PSUMMIT-2 trials. Ann Rheum Dis. 2014;73:1000-1006.

41. Podubbnyy D, Callhoff J, Listing J, et al. Ustekinumab for the treatment of patients with active ankylosing spondylitis: results of a 28 -week, prospective, open-label, proof-of-concept study (TOPAS). Arthritis Rheumatol. 2013;65(10 (Suppl)):S766.

42. Langley RG, Elewski BE, Lebwohl M, et al. Secukinumab in plaque psoriasis - results of two phase 3 trials. $N$ Engl J Med. 2014;371: 326-338.

43. van der Heijde D, Landewe R, Mease P, et al. Secukinumab, a monoclonal antibody to interleukin-17A, provides significant and sustained inhibition of joint structural damage in active psoriatic arthritis regardless of prior TNF inhibitors or concomitant methotrexate: a phase 3 randomized, double-blind, placebo-controlled study. Arthritis Rheum. 2014;66(Suppl):S424. Abstract 954. 
44. McInnes IB, Mease P, Kirkham B, et al. Secukinumab, a human anti-interleukin-17A monoclonal antibody, improves active psoriatic arthritis: 24-week efficacy and safety data from a phase 3 randomized, multicenter, double-blind, placebo-controlled study using subcutaneous dosing. Late breaking oral presentation at ACR/ARHP Annual Meeting, Boston, MA, USA, 2014. Presentation number L1.

45. Baeten D, Braun J, Baraliakos X, et al. Secukinumab, a monoclonal antibody to interleukin-17A, significantly improves signs and symptoms of active ankylosing spondylitis: results of a 52-week phase 3 randomized placebo-controlled trial with intravenous loading and subcutaneous maintenance dosing. Arthritis Rheum. 2014;66(Suppl):S360. Abstract 819.

46. Sieper J, Braun J, Baraliakos X, et al. Secukinumab, a monoclonal antibody to interleukin-17A, significantly improves signs and symptoms of active ankylosing spondylitis: results of a phase 3, randomized, placebo-controlled trial with subcutaneous loading and maintenance dosing. Arthritis Rheum. 2014;66(Suppl):S232. Abstract 536.
47. Mease PJ, Genovese MC, Greenwald MW, et al. Brodalumab, an antiIL17 RA monoclonal antibody, in psoriatic arthritis. $N$ Engl J Med. 2014;370:2295-2306.

48. Papp KA, Leonardi C, Menter A, et al. Brodalumab, an antiinterleukin-17-receptor antibody for psoriasis. N Engl J Med. 2012;366: 1181-1189.

49. Martin DA, Churchill M, Flores-Suarez L, et al. A phase Ib multiple ascending dose study evaluating safety, pharmacokinetics, and early clinical response of brodalumab, a human anti-IL-17R antibody, in methotrexate-resistant rheumatoid arthritis. Arthritis Res Ther. 2013;15:R164.

50. Pavelka K, Chon Y, Newmark R, et al. A study to evaluate the safety, tolerability, and efficacy of brodalumab in subjects with rheumatoid arthritis and an inadequate response to methotrexate. J Rheumatol. 2015;42:912-919.

\section{Publish your work in this journal}

ImmunoTargets and Therapy is an international, peer-reviewed open access journal focusing on the immunological basis of diseases, potential targets for immune based therapy and treatment protocols employed to improve patient management. Basic immunology and physiology of the immune system in health, and disease will be also covered. In addition, the journal will focus on the impact of manage-

\section{Dovepress}

ment programs and new therapeutic agents and protocols on patient perspectives such as quality of life, adherence and satisfaction. The manuscript management system is completely online and includes a very quick and fair peer-review system, which is all easy to use. Visit http://www.dovepress.com/testimonials.php to read real quotes from published authors.

Submit your manuscript here: http://www.dovepress.com/immunotargets-and-therapy-journal 